\title{
Research on the development patterns of sharing economy based on block chain technology
}

\author{
Luzhao Zhou ${ }^{1, ~ a, ~}{ }^{*}$, Qian Jia1, b \\ ${ }^{1}$ School of Economics and Management of Northwestern Polytechnical University Mingde College, \\ Xi 'an, shanxi, China \\ a20519959@qq.com, bjiaqianxd@163.com
}

Keywords: Sharing economy, Block chain, Application model, Development patterns.

\begin{abstract}
In the context of the coordinated development of mobile Internet and big data, block chain technology has promoted a new pattern of sharing economy, which realized the connection between supply and demand. And social resources are effectively and conveniently matched, so as to be fully utilized. However, enterprises based on the sharing economy will inevitably face problems such as lax supervision, unbalanced distribution of interests and hidden dangers in information security. This paper explains the connotation of sharing economy, four advantages of block chain technology and its application model, and then discusses the development model of sharing economy based on block chain technology. First, we need to build a sharing economy side chain governed by a user and function provider together. Second, we need to promote sharing economy using intelligent architecture. Third, we need to build mechanism of sharing economic data tracking and information security using timestamp technology. Finally, we need to construct a good sharing economy experience environment system, which uses trusted mechanism of security and anonymity.
\end{abstract}

\section{基于区块链技术的共享经济发展模式研究

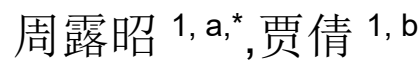 \\ 1 西北工业大学明德学院经济与管理学院, 西安, 陕西, 中国 a20519959@qq.com, bjiaqianxd@163.com}

关键词：共享经济, 区块链, 应用模型, 发展模式

中文摘要. 在移动互联网和大数据协同发展的背景下, 区块链技术催生了共享经济新模式, 实 现了供需双方的对接, 社会资源被有效便捷匹配, 进而被充分利用。然而以共享经济为基础 发展起来的企业, 具体运营中难免出现监管不严、利益分配不均衡、信息安全存在隐患等问 题。文中阐释了共享经济的内涵、区块链技术的四大优势及其应用模型, 进而探讨基于区块 链技术的共享经济发展模式。首先要建立用户、功能提供者共同治理的共享经济侧链, 其次 是运用智能合约架构助力共享经济新发展, 再次是运用时间翟技术构建用户共享经济数据追 踪与信息防伪机制, 最后运用安全与匿名的可信任机制构建良好的共享经济体验环境系统。

\section{1. 引言}

在我国综合国力不断提升的背景下，经济发展水平也随之持续上升，由过去的稀缺型经 济向过剩型经济变化, 而此时出现了许多物品并没有充分发挥本身应有价值的现象。那么闲 置物品集中一起实施共享理应是很好的选择, 可以说是互联网和大数据联合催生了共享经济。 
共享经济于 1978 年菲尔逊与斯潘思在私人汽车共享时提出, 简单地定义为在亲戚、朋友中进 行闲置资源使用, 为此提供一种便捷型服务。紧随其后 Zipcar 出现, Airbnb 在云计算十商业 智能的基础上应运而生，在此过程中以 Uber 为企业代表延伸至全球角落。顺应时代潮流我国 由此进入共享经济时期, 滴滴打车、58 同城、安居客、链家、瓜子二手车等企业迅猛发展起 来, 并融入日常生活。查阅相关文献和数据可知, 2017 全年我国国内在共享经济的基础上贸 易成交额为 49206 亿元, 同比增幅近 47.3\%。截至 2017 年底全球有 226 家独角兽企业, 仅我 国约 61 家，其中共享经济典型企业我国有 31 家，占国内独角兽企业的 $51.8 \%$ 。共享经济发 展在某种程度上增加了就业岗位, 也有利于包容性增长, 我国逐渐成为世界共享经济的领跑 者。在其发展过程中, 我们依然发现国内的共享经济处于初期阶段难免存在一些迫切需要解 决的问题, 为此, 出现了一批学者着手对区块链运用到共享经济中进行探究。区块链原理最 早始于中本聪的“点对点电子货币体系”观点, 而吸引学者研究的就是区块链去中心化、信息 无法篡改等特点, 并结合现阶段已有的文献给出适应地运营模式和相关运营体系。本文就这 一问题结合“区块链+共享经济”新模式解决其发展过程中所存在的监管不严、专权垄断、信息 隐患等系列问题, 助推共享经济的可持续发展。

\section{2. 共享经济的内涵}

截止当前对共享经济的概念各有所依，并没有就此达到一个共识的概念。笔者基于国外 多数学者的观点总结共享经济的概念, 点对点交易模式即资源拥有者同资源需求者以“互联网 +大数据”为媒介搭建互利双赢的模式。例如 2007 年 Belk 所给出的观点“高效+大规模+点对点” 的共享经济, 借助互联网来壮大自身发展; 2014 年 De Grave 所给出的观点“生产分布式+融资 +点对点”的共享经济, 将其作为经济的后补方式; 2013 年以 Dervojeda 为首的数位学者所给 出的观点“商业模式+点对点”的共享经济, 而且具有可访问性, 并且时段内拥有资源者与需要 该资源者通过合理、可接受的经济成本来进行两者匹配；2015 年以 Hamari 为首的数位国外 学者所给出的观点 “通信技术+数类结合”共享经济, 此过程中将互联网作为基础。供给层面来 看, 产能剩余的情况出现, 此时的观点就是共享经济是一中转站, 以此进行资源在此分配来 换取相应的收益; 2014 年 Parsons 所给出的观点“产能+网络技术+组织或是个人”形成共享经 济进行产品循环式利用; 在 2015 年以 Olson 为首的国外多位学者所给出的观点就是“互联网+ 技能+分享”的模式，用户将可以有直观收益。

国内和国外相同也是在点对点和供给层面进行定义。一种角度将共享经济看作点对点产 品交易模式，2017 年学者丹所给观点是共享经济营运主体是以“组织对个人、个人对个人”模 式, 也可理解成点对点的交易模式; 2017 年郑云坚为首的多位学者所给出的观点是共享经济 可以与点对点交易划等号, 点对点交易就等同于共享经济; 2015 年学者孟凡新所给出的观点 是把个体对个体的商品交换或者服务交换的消费模式比作共享经济。另一种角度是基于供给 侧来定义共享经济。2016 年学者杨帅所给出的观点是依托互联网技术将组织或是个人的闲置 资源及服务产品进行交换和共享模式; 2016 年以李波为首的多位学者所给出的观点是人和人 之间依托第三方互联网平台实现过剩产能、服务交换的商业模式; 2017 年以王芳为首的数位 学者所给出的观点是帮助社会上的过剩产能进行二次配置来获取相应的收益, 并且将资源使 用率进一步提升。

\section{3. 区块链技术的四大优势及其应用模型}

分布式数据存储技术在区块链中体现出, 存储及验证数据依托块链式结构, 基于分布式 节点产出共识算法和数据同步并且永续存在、信息不可发生改变（篡改）, 一直保存在区块 链里。对全员信息录入，链内信息发生变动时区块链也随之进行延展。 


\section{1 区块链技术的四大优势}

去中心化成为区块链技术的核心优势，以数据加密、分布式为其显著特征。在分布式系 统中不需要互信存在于节点, 依旧可以将点对点交易实现, 有效解决高成本、效率不高、信 息存储遗漏等问题。而作为网络协议的区块链技术，这一技术应用优势有这样几类:

\section{1 .1 去中心化及分布式记录与存储可节省存储中介成本}

区块链中的关键一环是去中心化，它的实现依赖以下三个维度：一是去中心化的组织层、 二是去中心化的权控层、三是共识机制的逻辑层。组织系统少故障是基于去中心化的组织层 和去中心化的权控层, 单一节点不能进行完全系统控制, 但在逻辑层面需要群体达成共识, 之所以去中心化系统有存在的可能性也正是因为有共识机制。同时, 世界范围内信息均在区 块链中呈现出分布式记录与存储, 其信息均能保持更新的进度, 不间断运行前提条件是网络 不全系统崩溃。因此，区块链技术可有效规避中心化数据库技术容易引发集体崩溃的核心缺 陷, 同时, 去中心化的架构方式将成本缩减, 即不需要一个中心存储系统来存储所有数据。

3.1 .2 可编程的智能合约有利于规范用户行为

将有价值的信息进行选择性地存储是可编程的智能合约灵活形式体现方式，根据用户的 需求给出两个私钥, 也可以给出几个私钥, 甚至不要任何的私钥等灵活形式来服务用户。或 者在将价值信息发送时, 附加价值转移的条件, 对价值信息进行用途规范。综上来看去中心 化的环境是需要其全部协议均有所共识, 其优势还有智能合约将区块链中系统解决交易未来 方式得以实现，其技术运用将同步更新，实用性完整体现。

\section{1 .3 不可篡改的时间翟技术有利于数据追踪与信息防伪}

区块的生成取决于系统，一般而言区块在几分钟就可以形成，并且每个形成的区块均有 连接点 ID, 可以有效区分出区块的前后区域界定，十分容易地找到起始节点。同时，块链中 均有时间翟记录, 时间起始点都一目了然, 并且具有不可篡改和不可伪造的数据, 将程序进 行了优化，简便办事流程。拥有时间翟，既可将某人在某天确实做过某事进行真实记录，还 可以明确活动的发起者, 通俗来讲时间翟, 就是公证人的角色, 相比传统其公信力更高, 对 数据的追踪和信息的安全提供了全程保障。

\section{1 .4 安全与匿名的可信任机制有利于节省信用中介成本}

为更好地解决共识机制将“非对称加密数学”的密码学原理引用到区块链，简单来讲就是 加密和解密不共用一个密码, 而且全体均可用自己的公钥加密一段信息 (确保信息的真实性), 这一做法就是要在解密的时候需要自己的私钥解开（确保信息的安全性）。节点记录结果的 正确性依托区块链协议机制, 其要求就是多数节点要结果相一致此时真实性才会被认可, 数 据方面也就自然被写入其中。该项技术刷新了以往信用方式, 即是参与前无需对背景进行了 解, 也无需第三方机构进行保障, 此项保障对价值转移实施记录、存储、传输等得到公认的 结果, 将物联信用中介数量减少, 同时也是对物联网需要信用中介这一缺陷的弥补, 既能保 持信息的安全又能将成本有效性缩减。

当前, 去中心化区块链逐渐向公共区块链方向变化, 出现对公有侧链的现象。区块链的 雏形就是公共区块链, 它是一种整过程去中心化的阶梯型存储, 在访问上不受限制尤其是公 共块链中的相关数据, 在进行价值交换时信任机制借助密码学相应技术对此加以保障。公共 区块链由内向外延伸的过程就是侧链的发展, 此时的过程相当于一个桥梁的作用, 将公共区 块链和其他价值进行多区块链转移，而且在对信用证明时，侧链能支持数据结构的复杂处理。

区块链技术的应用渐渐展开，相应平台多次尝试借助区块链平台进行版权流程保护和多 方式的内容发布，比如百度服务平台即是最好的体现方式，再比如，2017 腾讯白皮书提到区 块链的整体架构有了完全的呈现, 而且腾讯区块链模式已经在金融、数字资产、物流上运用。

\section{2 区块链技术的应用模型}

从价值取向角度来说，人们所认同的共识意见是区块链的一种基础，那么我们就可以认 
为区块链中跨链的本质是一种等价交换和交易平台。方便读者对此有个更加清楚地了解, 笔 者将模型简化叙述，架构图如下:

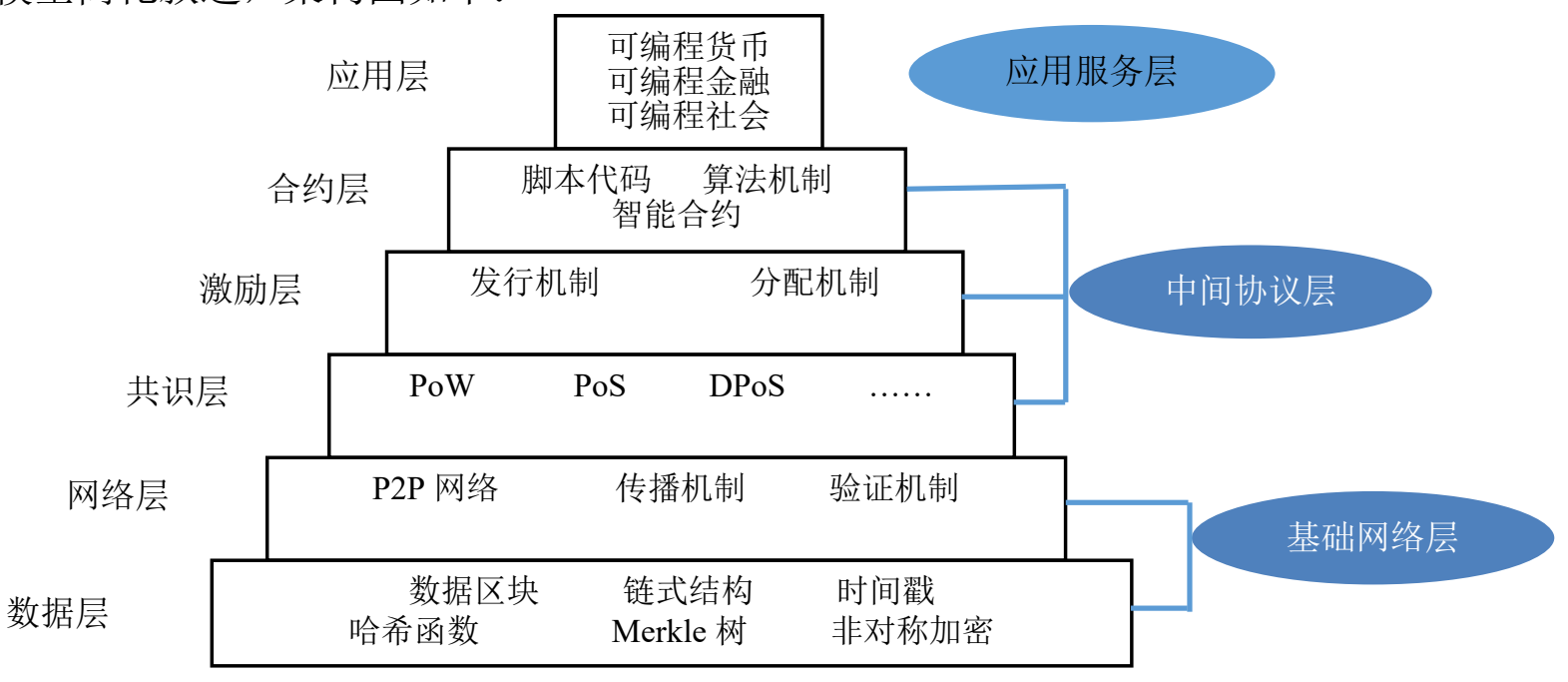

图 1: 区块链的模型架构

由图 1 可直观看出区块链 6 层架构模型:

（1）数据层: 作为块链的基础层，对数据架构加以封装，时间戳技术和对非对称的公钥 及私钥进行加密。

(2) 网络层: 组网机制是以点对点形成，介于这一特性进行数据的传播以及数据校验, 并且具有组网自动性，通常被看做分布式自治模式（DAOs）。

（3）共识层：机制的共识、算法集中在这一层面，一般情况下有三类常见共识机制：算 法 pow、权益证明机制 pos、股份授权证明机制 Dpo。此三类具有利弊伴随左右，是一个曲折 前进的阶段。

(4) 激励层: 该层发行和分配激励即是奖励层面，也是工资系统。对此理解我们要对比 特币进行了解, 即可以这样理解：区块新领域全新奖励+交易所需费用或是奖励+手续费过程 分配成为该层的功能。

（5）合约层：区块具有编程、代码的嵌入所具有的特点, 并且涵盖脚本、算法和智能合 约, 简单理解成电子合同, 它具备了达到条件智能履行, 人工干预极少甚至不存在人工干预; 在不满足时其合约智能解除，这一层面可谓是信用体系中的核心技术之一。

（6）应用层：同以往的网络协议模型大致相同，也是对各类场景和案例的加装，和日常 所用网站和 APP 相同或者比较类似，如电商娱乐和新闻阅读及其他场景的运用。

通过对架构模型的认知和了解，我们可以看出在区块链的各层面均会有新岗位出现或者 新的创业机遇, 并且有可能成为行业领跑者。并且在应用和合约层对人才需求增多成为一种 趋势, 智能合约最为突出的现象就是 ETC 的综合运用对此有了清晰的范本, 而比特币的运营 则是应用层的典型代表，接下来由笔者带领读者看区块链技术新趋势的三个阶段，在其发展 过程中出现了许多权威论证进行明确领域，多数情况下成了共识:
A、区块链 1.0 阶段 数字货币（可编程货币）
B、区块链 2.0 阶段 智能合同（可编程金融）
C、区块链 3.0 阶段一一高级智能合同（可编程社会）

集类型、功能的区块链要求, 和趋势走向及架构模型, 读者可以将高阶段作为参考来构 思所要设计的区块链或者进行开发参考, 借用本国互联网的优势将可以实现区块链为社会生 产所带来的变革新力量。 


\section{3 区块链技术在共享经济发展中的应用}

\subsection{1 信任机制的建立}

区块链网构里, 不被指定一个网络节点和另一个网络节点实施数据间交换时不再建立事 前互信任，仅仅需要两者进行交易的时候采用数字化办公技术对相应身份加以验证，由此可 以看出, 现阶段共享经济中不需要将押金或者是检索信用积分等信任认证机制, 但要求进行 数据交换时依据系统所设定的规则既可, 确保了网构中一个节点和另外一个节点彼此间的信 任而不出现欺骗。换句话来说, 减少交易成本费用, 就是要将区块链技术运用到共享经济中 进行两者信任的搭建，共同实现信任机制的建立。

\subsection{2 数据安全和存储可追溯}

保障数据的真实性是区块链技术一个显著的特征，在综合运用数字化摘要、签名和非对 称加密时, 保障了数据的全过程传输以及所有节点均处于最稳定的网络环境之中, 有效进行 数据的保护。用户信息之所以安全有保障是源于公钥、私钥的出现有了隐匿性及隐蔽性。在 这里需要说明用户可以拥有多个公钥或者多个私钥, 基于生成多个公钥或是私钥服务一个用 户群体时, 服务过程中的公钥均有一个全新的身份进行代表, 就用户隐私问题进行防御保护, 形成了以技术为核心的数据保护，促使数据的持续保存。

\subsection{3 组建新型共享模式}

资源的优化是基于共享经济的推动，在区块链技术为基础的情况下共享经济的实际价值 将会在原有的基础上再次被增值，由过去分散型使用到现在的集中型使用真正实现了杂乱无 章的经济进入综合利用的共享经济，其价值得到了有效发挥，同时也实现了全民共享。

以 P2P 网络为结构依撑的区块链，两者间在享受共享服务的同时有了更宽阔的领域，阶 段内只要存在过剩的资源, 均可在网构中的其他节点上进行资源共享服务, 进一步实现了供 需关系由买卖方决定市场份额。区块链技术对其物品进行公证, 源于共识机制的创建, 对过 程中授权信息和认证信息进行了全程防御。对上述情况有所了解的基础上，区块中就会被载 入共享资源使用者的用户评价信息，自然形成区块中评价机制，实现优质服务与经济利益相 挂钩, 以此参与共享经济所带来的优质服务。尤其需要注意的是, 降低经济成本助推利益最 优化是区块链的综合表现。

\section{4. 基于区块链技术的共享经济发展模式}

结合区块链技术发展共享经济，核心思路是借助区块链技术将共享经济由各类共享经济 APP“各自为政”的松散架构整合为内容、形式、功能多样化的共享经济“共同体”，搭建脱离中 心服务体系，既能用户间直接沟通，也能用户和研发者进行交换，进一步改善用户共享经济 体验，提升用户共享经济效果，同时，运用区块链技术的共识机制加强政府等组织机构对共 享经济推广的管理，帮助相关机构开展线上线下活动组织，进一步推广共享经济。

\section{1 建立用户、功能提供者共同治理的共享经济侧链}

新技术既能使用户转变生产者，也能决定其技术使用方式，同时，网络系统的终极价值 在于该系统对用户有什么好处, 由用户和功能提供者共同治理共享经济系统, 有利于确保双 方的利益均能得到实现，因此，建立用户、功能提供者共同治理的共享经济侧链十分重要。

用户、功能提供者共同治理的共享经济侧链要求将所有共享经济类 APP 以链的方式组合 在一起, 结合区块链已有结构构造共享经济类 APP 侧链, 每一个共享经济类 APP 都不会成 为侧链的中心和控制权的中心，但它们需要达成一个共识以保证该侧链的正常运行，这个共 识包括对用户、功能提供者的行为规范的共识，如功能提供者之间制定协议确保用户可以在 不同共享经济类 APP 之间跨平台执行阅读、消费等操作。同时，历史数据均可由侧链存储， 用户与用户、用户与功能提供者、功能提供者之间每一次交互的数据都可以被记录下来。保 
障数据存储在侧链中的同时进行持续刷新, 构建一个可以落地的共享经济侧链应用场景, 用 户和用户在这个场景中进行阅读分享、互赠书籍及书币等行为, 用户和功能提供者在这个场 景中实施交流、交易等行为, 多个功能提供者在这个场景中共同为用户提供各种个性化服务, 如允许书币互换等行为, 实现用户、功能提供者自治的同时, 满足用户各类共享经济需求, 确保功能提供者的商业利润最大化。

\section{2 运用智能合约架构助力共享经济新发展}

合同的新发展阶段就体现在区块链智能合约，通常是以纸质合同、电子合同、数字合同 三类为外部载体的合同分类。简单来说就是纸质合同最为古老，电子合同也仅仅是运用信息 关联技术，这两种合同并没有本质性的差别。伴随时代“信息技术+计算机技术”的发展变化， 电子合同便不能满足了当前的需要，紧随其后诞生了数据合同。该合同由计算机系统进行协 议或是条款的预设, 另外还出现可计算合同即由系统进行数据赋值, 并且自动将合约或是履 行实现个性评估。换而言之就是在合同谈判和履行以及强制过程中计算机系统代替人工执行 相关操作。现阶段的区块链智能合同属于高阶段评判系统, 当到达预设事件时其本身就能够 快速完成, 有效将人工效用成本降低, 此外就是服务快捷。就比如滴滴专车, 用户有需求时 进入软件后输入自己的起始点, 由后台信息平台对这一交易进行合同的初期审理, 在进行就 近车源接驾, 到达目的系统进入自动结算费用, 待完成结算后整个流程全部走完, 然后系统 平台进行合同的最终校验。相比过去的纸质和电子合同在周期缩短的同时，也加快了共享经 济的均衡发展。

例如, Origin Protocol 以“太坊+IPFS”为基础, 实现任何人均可以轻松创建点对点的商品 服务, 而在共享数据层结合太坊区块链建立用户数字身份, 进行智能合约的执行, 在此过程 中将数据存储在 IPFS 上, 实现降低成本和保持链外的信息稳定性, 并且实现了应用平台的可 扩展性。

\section{3 运用时间唯技术构建用户共享经济数据追踪与信息防伪机制}

当前时间翟可划分为可信和非可信时间翟技术，笔者以前者技术为主运用在共享经济电 子数据记录中, 共享经济电子数据所涉及的领域比较多, 普遍存在的现象是数据内容被修改, 其内容的真实性存疑。随着时间翟的出现，将共享经济电子数据同时间翟技术相结合。

首先，对用户数据真实性有了很好的保障。其原因是用户数据形成之后是可以和依托的 载体相分离, 此过程中用户数据信息就会容易被修改, 信息就会出现不稳定的现象。为此用 户数据信息不能仅仅依靠后期的纸质信息来进行综合判断，而将时间翟技术运用其中，一定 程度上把以上现象得到有效解决。也可以这样理解, 在用户数据信息生成前使用时间嬥技术, 其内容将持续保持原样。

其次，用户数据多了有效性。充分利用时间戳技术使得用户数据上出现电子签名并出现 时间翟, 这一简单举措可以方便查出用户的相关信息和准确的记录时间, 充分进行了数据保 障和信息的真伪验证。

再次，用户数据的整体性。首先对整体性进行一个界定：用户信息不做任何修改，就说 明用户信息具有整体性。将时间翟技术与用户信息相结合并不会因此改变其中的内容，仅仅 作为一种历史凭证来使用，用户信息的稳定性就是源于时间翟中固化的作用，减少或是避免 用户信息发生变化从而确保信息的整体性。

\section{4 运用安全与匿名的可信任机制构建良好的共享经济体验环境系统}

在 Internet 普及的情况下，传统的互联网客户端限制网络系统资源的充分使用，对新形 势下的需求不能给予对应的满足，伴随着需求增长 P2P 网络应运而生， P2P 在开展技术间合 作、共享资源和大范围计算等方面出现显著的独到优势。

Peer-to-Peer 网络又称为对称网络, 是 Internet 网络的一个延伸, 是信息交换的一种模式 
也是一种网构思想。由于 P2P 网络中也有真实生活的写入，可以实现和人进行交流的网络环 境, 自然也对任何人都开放权限, 带来便利的同时也伴随着隐患的出现。比如：网络资源不 确定、木马及病毒的不定时传播、存疑的服务、无法保证信息都是正确的等。由此看来，以 上现象的出现正是由于没有可信任的机制加以控制而产生的。而此时 Internet 中的匿名成为了 信息共享的一种手段，通过一定的方式进行信息的加密处理，对相应的信息流加以隐藏，有 效阻止了对网络攻击者获取信息的途径，如基于区块链下共享经济在进行交易时可以将个人 的 IP 地址隐藏, 保护自己的隐私。从而也就构建了可信任机制, 更好地体验共享经济环境所 带来的优势。

\section{5. 结束语}

新时代的形势下区块链技术的发展是前所未有，结合它自身所具备的优势在共享经济加 以充分运用，帮助其系列问题的解决。举例：不对称的信息和不均衡的分配问题，经过区块 链技术对共享经济中存在问题的解决, 减少了共享资源平台互信成本的支出。因此以区块链 技术为基础联合共享经济，两者有效结合就形成了存续增值关系，此种经济的共享作为新型 模式，对现阶段内已有的共享经济形式形成冲击，营造出区块链下共享经济新局面。以区块 链技术为基础, 建立用户、功能提供者共同治理的共享经济侧链，运用智能合约构建规范的 共享经济行为体系，运用时间翟技术构建用户共享经济数据追踪与信息防伪机制，构建良好 的共享经济环境体验系统, 充分发挥区块链技术的去中心化、分布式、安全、智能的优势, 对发展我国共享经济意义重大。

关于借助区块链技术构建共享经济发展模式不能忽略的问题在于：一方面，我们在使用 任何技术时，会逐渐习惯依靠这种技术; 另一方面，基于区块链技术构建的共享经济推广新 模式在节约中心化成本时势必会造成过度使用电子能耗的状况。基于区块链技术构建的共享 经济发展新模式在我国共享经济发展方面也将发挥更大的作用。

\section{References}

[1] Rongshan Shi, Dan Li. Research on the development patterns of sharing economy based on block chain technology [J]. Business Economic Research. 2018(12): 178-181.

[2] Haiying Liu. Research on the development of "big data + block chain" sharing economy -based on the theory of industrial integration [J]. Technical Economy and Management Research. 2018(1):91-95.

[3] Runxiang Jiang, Changjiang Wei. Application progress and value discussion of block chain[J]. Gansu Province Financial. 2016(02): 19-21.

[4] Rongrong Zhou. Research on the development path and countermeasures of sharing economy based on block chain technology [J]. Guangxi Social Sciences. 2018(2): 84-87.

[5] Belk R. Why not share rather than own?[J]. Annals of the American Academy of Political and Social Science, 2007, 611(1).

[6] Hamari J, Sjöklint M, Ukkonen A. The Sharing Economy: Why People Participate in Collaborative Consumption[R]. 2015.

[7] Bardhi, Fleura, Eckhardt, Giana M. Access-Based Consumption: The Case of Car Sharing [J]. Journal of Consumer Research. 2012.

[8] Fleura Bardhi, Giana M. Eckhardt. Access- based Consumption: The Case of Car Sharing [J]. Journal of Consumer Research, 2012.

[9] James. Blockchain: Putting Theory into Practice [J]. Schneider, 2016. 
[10] Bughin, Jacques, Livingston, John, Marwaha Sam. Seizing the Potential of "BigData"[J]. MckinseyQuarterly, 2011.

[11] M Waterson. Regulation of the Firm and Natural Monopoly [J].Economic Journal, 1989, 99(394).

[12] R. Amit, C. Zott. ValueCreationinE-Business[J]. StrategicManagementJournal, 2001(22).

[13] KK Fung, SH Kolar, P Karnam . Regulating Natural Monopoly: Flash Animation[J]. Journal of Economic Education, 2007, 38(2).

[14] Felson M, SPAETH J L.Community Structure and Collaborative Consumption[J]. American Behavioral Scientist, 1978, 21(4).

[15] Lamberton C P.Rose R L. When is ours better than mine?A framework for understanding and altering participation incommercial sharing systems[J]. Journal of Marketing, 2012, 76(04): 109-125. 